\title{
Motion Caused by Magnetic Field in Lobachevsky Space
}

\author{
V.V. Kudryashov, Yu.A. Kurochkin, E.M. Ovsiyuk and V.M. Red'kov \\ B.I. Stepanov Institute of Physics, \\ National Academy of Sciences of Belarus, \\ 68 Nezavisimosti Ave., 220072 Minsk, Belarus
}

\begin{abstract}
We study motion of a relativistic particle in the 3-dimensional Lobachevsky space in the presence of an external magnetic field which is analogous to a constant uniform magnetic field in the Euclidean space. Three integrals of motion are found and equations of motion are solved exactly in the special cylindrical coordinates. Motion on surface of the cylinder of constant radius is considered in detail.
\end{abstract}

PACS numbers: $02.40-\mathrm{k}, 03.50-\mathrm{z}$

Keywords: Lobachevsky space, magnetic field, Lagrange equations

\section{INTRODUCTION}

The description of a particle behavior in a constant uniform magnetic field is one of the basic problems in theoretical physics. As well known in the case of the Euclidean space, this problem is exactly solved in the classical [1] and quantum [2,3] mechanics. Recently the generalization of the quantum mechanical solution to the case of the Lobachevsky space was given [4,5].

In the present paper, an exact description of a relativistic motion of a classical particle in an external magnetic field will be constructed on the background of the hyperbolic Lobachevsky $H_{3}$ space. This result can be used to describe the behavior of the charged particles in macroscopic magnetic field in the context of astrophysics.

\section{THE LAGRANGE EQUATIONS AND INTEGRALS OF MOTION}

We start with a metric of the Lobachevsky space in the special cylindrical coordinates $[6]$ :

$$
\begin{array}{r}
d S^{2}=c^{2} d t^{2}-\cosh ^{2}(z / \rho) d r^{2} \\
-\rho^{2} \cosh ^{2}(z / \rho) \sinh ^{2}(r / \rho) d \phi^{2}-d z^{2}, \\
z \in(-\infty,+\infty), \quad r \in[0,+\infty), \quad \phi \in[0,2 \pi],
\end{array}
$$

where $\rho$ is a curvature radius. The curvilinear coordinates $(r, \phi, z)$ of the three-dimensional Lobachevsky space are connected with quasi-Cartesian coordinates of the comprehending four-dimensional pseudo-Euclidean space by relations

$$
\begin{array}{r}
u_{1}=\rho \cosh (z / \rho) \sinh (r / \rho) \cos \phi, \\
u_{2}=\rho \cosh (z / \rho) \sinh (r / \rho) \sin \phi, \\
u_{3}=\rho \sinh (z / \rho), \\
u_{0}=\rho \cosh (z / \rho) \sinh (r / \rho), \\
u_{0}^{2}-u_{1}^{2}-u_{2}^{2}-u_{3}^{3}=\rho^{2}, \quad u_{0}=+\sqrt{\rho^{2}+\mathbf{u}^{2}} .
\end{array}
$$

The given metric determines the explicit expression for a squared particle velocity

$$
\begin{gathered}
\epsilon=\left(\frac{d z}{d t}\right)^{2}+\cosh ^{2}(z / \rho)\left(\frac{d r}{d t}\right)^{2} \\
+\cosh ^{2}(z / \rho) \rho^{2} \sinh ^{2}(r / \rho)\left(\frac{d \phi}{d t}\right)^{2} .
\end{gathered}
$$

In accordance with $[4,5]$ we define a potential for a magnetic field in the following form

$$
A_{\phi}=-\rho^{2} B[\cosh (r / \rho)-1] .
$$

This potential generalizes a potential for a constant uniform magnetic field with a strength $B$ in the Euclidean space when we choose the direction of the field as the $z$ axis and use the usual cylindrical coordinates. The potential (3) is the solution of Maxwell's equations in the Lobachevsky space. Here these equations are reduced to a single equation

$$
\frac{1}{\sqrt{-g}} \frac{\partial}{\partial x^{r}} \sqrt{-g} F^{r \phi}=0
$$

where

$$
\begin{array}{r}
F_{\phi r}=\partial_{\phi} A_{r}-\partial_{r} A_{\phi}, \\
\sqrt{-g}=\cosh ^{2}(z / \rho) \sinh (r / \rho) .
\end{array}
$$

The relativistic Lagrangian for a particle in above magnetic field is of the form

$$
\begin{array}{r}
L=-m c^{2} \sqrt{1-\frac{\epsilon}{c^{2}}} \\
+\frac{e B \rho^{2}}{c}[\cosh (r / \rho)-1]\left(\frac{d \phi}{d t}\right) .
\end{array}
$$

Then the Lagrange equations of the second order are expressed as

$$
\begin{gathered}
\frac{d^{2} r}{d t^{2}}+2 \tanh (z / \rho)\left(\frac{d z}{d t}\right)\left(\frac{d r}{d t}\right)=\rho \sinh (r / \rho) \\
\times\left[\cosh (r / \rho)\left(\frac{d \phi}{d t}\right)+\frac{\omega}{\cosh ^{2}(z / \rho)}\right]\left(\frac{d \phi}{d t}\right),
\end{gathered}
$$




$$
\begin{array}{r}
\frac{d}{d t}\left[\rho^{2} \sinh ^{2}(r / \rho) \cosh ^{2}(z / \rho)\left(\frac{d \phi}{d t}\right)\right. \\
\left.+\omega \rho^{2}(\cosh (r / \rho)-1)\right]=0 \\
\quad \frac{d^{2} z}{d t^{2}}-\frac{1}{\rho} \cosh (z / \rho) \sinh (z / \rho) \\
\times\left[\left(\frac{d r}{d t}\right)^{2}+\rho^{2} \sinh ^{2}(r / \rho)\left(\frac{d \phi}{d t}\right)^{2}\right]=0 .
\end{array}
$$

Here we have introduced notation for a quantity

$$
\omega=\frac{e B}{m c} \sqrt{1-\frac{\epsilon}{c^{2}}} .
$$

The squared velocity $\epsilon$ is the integral of motion for the considered system in the Lobachevsky space as the usual squared velocity is the integral of motion in the Euclidean space.

It is obvious that a quantity

$$
\begin{array}{r}
I=\omega \rho^{2}[\cosh (r / \rho)-1] \\
+\rho^{2} \sinh ^{2}(r / \rho) \cosh ^{2}(z / \rho)\left(\frac{d \phi}{d t}\right)
\end{array}
$$

is conserved. This integral of motion is the generalization of the usual angular momentum.

At last it is simply to verify that in addition to the conserved quantities $\epsilon$ and $I$, third quantity

$$
A=\cosh ^{4}(z / \rho)\left[\left(\frac{d r}{d t}\right)^{2}+\rho^{2} \sinh ^{2}(r / \rho)\left(\frac{d \phi}{d t}\right)^{2}\right]
$$

is the integral of motion, at $\rho \rightarrow \infty$ it tends to the squared velocity of motion perpendicular to $z$ axis.

\section{INTEGRATION OF THE EQUATIONS OF MOTION}

It should be stressed that the use of the special cylindrical coordinates allows us to find the simple analytical expressions for three integrals of motion. In the presence of these conserved quantities it is easily to perform transition from the initial equations of the second order (5) (7) to the following equations of the first order:

$$
\begin{gathered}
\left(\frac{d z}{d t}\right)^{2}=\epsilon-\frac{A}{\cosh ^{2}(z / \rho)} \\
\left(\frac{d r}{d t}\right)^{2}=\frac{A}{\cosh ^{4}(z / \rho)}-\frac{\left[I-\omega \rho^{2}(\cosh (r / \rho)-1)\right]^{2}}{\cosh ^{4}(z / \rho) \rho^{2} \sinh ^{2}(r / \rho)},
\end{gathered}
$$

$$
\frac{d \phi}{d t}=\frac{I-\omega \rho^{2}(\cosh (r / \rho)-1)}{\rho^{2} \sinh ^{2}(r / \rho) \cosh ^{2}(z / \rho)} .
$$

First of all we consider Eq. (12). The sign of the quantity $\epsilon-A$ determines the character of solution.

If $\epsilon>A$, then we have the explicit expressions for the coordinate

$$
\sinh (z(t) / \rho)= \pm \sqrt{1-\frac{A}{\epsilon}} \sinh \left(\sqrt{\epsilon}\left(t-t_{0}\right) / \rho\right)
$$

and the velocity

$$
\frac{d z(t)}{d t}= \pm \sqrt{\epsilon-\frac{A \epsilon}{(\epsilon-A) \sinh ^{2}\left(\sqrt{\epsilon}\left(t-t_{0}\right) / \rho\right)+\epsilon}} .
$$

In this case a particle passes through the point $z=0$ with velocity $d z / d t= \pm \sqrt{\epsilon-A}$.

If $\epsilon<A$, then we have other explicit expressions for the coordinate

$$
\sinh (z(t) / \rho)= \pm \sqrt{\frac{A}{\epsilon}-1} \cosh \left(\sqrt{\epsilon}\left(t-t_{0}\right) / \rho\right)
$$

and the velocity

$$
\frac{d z(t)}{d t}= \pm \sqrt{\epsilon-\frac{A \epsilon}{(A-\epsilon) \sinh ^{2}\left(\sqrt{\epsilon}\left(t-t_{0}\right) / \rho\right)+A}}
$$

In this case the particle moves either in the region

$$
\sinh (z / \rho)>\sinh \left(z_{+} / \rho\right)=\sqrt{A / \epsilon-1}
$$

or in the region

$$
\sinh (z / \rho)<\sinh \left(z_{-} / \rho\right)=-\sqrt{A / \epsilon-1} .
$$

In other words there is the repulsion at the points $z_{-}$ and $z_{+}$leading to existence of the forbidden region for motion.

It should be noted that velocity along $z$ axis is constant in the case of the Euclidean space $(\rho \rightarrow \infty)$ whereas velocity along $z$ axis is variable in the case of the Lobachevsky space.

From the equations (12) - (14) it is seen that after obtaining the explicit dependence $z(t)$, the further solution of the problem is reduced to the following integrations

$$
= \pm \int \frac{\int \frac{\rho \sinh (r / \rho) d r}{\sqrt{w(r)}}}{\cosh (z / \rho) \sqrt{\epsilon \cosh ^{2}(z / \rho)-A}}
$$




$$
\phi-\phi_{0}= \pm \int \frac{\left[I-\omega \rho^{2}(\cosh (r / \rho)-1)\right] d r}{\rho \sinh (r / \rho) \sqrt{w(r)}}
$$

where

$$
w(r)=A \rho^{2} \sinh ^{2}(r / \rho)-\left[I-\omega \rho^{2}(\cosh (r / \rho)-1)\right]^{2} .
$$

Both formula (19) for the dependence $r(z)$ and formula (20) for the dependence $\phi(r)$ give the particle orbit.

Now we examine the connection of $\phi$ and $r$ in detail. The integration in (20) leads to the following relation

$$
\begin{aligned}
& \left(I+\omega \rho^{2}\right) \cosh (r / \rho)-\omega \rho^{2} \\
= & \sqrt{C} \rho \sinh (r / \rho) \cos \left(\phi-\phi_{0}\right),
\end{aligned}
$$

where

$$
C=A-\rho^{2} \omega^{2}+\frac{1}{\rho^{2}}\left(I+\omega \rho^{2}\right)^{2} .
$$

A quantity $C$ as function of $A$ and $I$ is the integral of motion and has the form

$$
\begin{gathered}
C=\cosh ^{4}(z / \rho)\left(\frac{d r}{d t}\right)^{2}+\rho^{2} \sinh ^{2}(r / \rho) \\
\times\left[\cosh (r / \rho) \cosh ^{2}(z / \rho)\left(\frac{d \phi}{d t}\right)+\omega\right]^{2}, \\
C \geq 0 .
\end{gathered}
$$

\section{THE MOTION ON THE CYLINDRICAL SURFACE}

Let us compare formula (21) with the well known formula

$$
\begin{array}{r}
\cosh (R / \rho) \cosh (r / \rho)-\cosh \left(r_{0} / \rho\right) \\
=\sinh (R / \rho) \sinh (r / \rho) \cos \left(\phi-\phi_{0}\right)
\end{array}
$$

describing in the Lobachevsky space a circle of radius $r_{0}$, if a distance between a circle center and the origin of coordinates is $R$. Formulas (21) and (24) coincide, if the parameters $R$ and $r_{0}$ are connected with the integrals of motion $A$ and $I$ by the relations

$$
\begin{gathered}
\cosh \left(r_{0} / \rho\right)=\frac{1}{\sqrt{1-A / \omega^{2} \rho^{2}}}, \\
\cosh (R / \rho)=\frac{I+\omega \rho^{2}}{\omega \rho^{2} \sqrt{1-A / \omega^{2} \rho^{2}}} .
\end{gathered}
$$

We emphasize that the expressions (25) and (26) become sensible, if the inequality

$$
A<\omega^{2} \rho^{2}
$$

is accomplished. Then in the $(r, \phi)$ - projection, a particle moves along the circle. The circle center is shifted with respect to the origin. Taking into account a motion in the $z$ direction we get a complete motion on the cylindrical surface. The coincidence of a coordinate origin and a circle center corresponds to the zeroth value of the integral of motion $C$. In this case a $z$ axis coincides with a cylinder axis.

The transition to this coordinate system leads to the considerable simplification. From the condition $C=0$ we see that

$$
\begin{gathered}
\frac{d r}{d t}=0, \quad\left(r=r_{0}\right), \\
\frac{d \phi}{d t}=-\frac{\omega}{\cosh \left(r_{0} / \rho\right) \cosh ^{2}(z / \rho)} .
\end{gathered}
$$

In this case the integrals of motion $A$ and $I$ are expressed via the circle radius $r_{0}$ as follows

$$
\begin{gathered}
A=\omega^{2} \rho^{2} \tanh ^{2}\left(r_{0} / \rho\right), \\
I=\omega \rho^{2}\left[\frac{1}{\cosh \left(r_{0} / \rho\right)}-1\right] .
\end{gathered}
$$

Taking into account the relations (15) - (18) we get the explicit formulas describing evolution of an angle and an angular velocity.

If $\epsilon>A$, we obtain the following expressions

$$
\begin{gathered}
\phi(t)-\phi_{0}=\frac{-\omega \rho}{\cosh \left(r_{0} / \rho\right) \sqrt{A}} \\
\times \operatorname{artanh}\left[\sqrt{\frac{A}{\epsilon}} \tanh \left(\sqrt{\epsilon}\left(t-t_{0}\right) / \rho\right)\right], \\
\frac{d \phi(t)}{d t}=\frac{-\omega \epsilon}{\cosh \left(r_{0} / \rho\right)\left[(\epsilon-A) \sinh ^{2}\left(\sqrt{\epsilon}\left(t-t_{0}\right) / \rho\right)+\epsilon\right]}
\end{gathered}
$$

and if $\epsilon<A$, we obtain other expressions

$$
\begin{array}{r}
\phi(t)-\phi_{0}=\frac{-\omega \rho}{\cosh \left(r_{0} / \rho\right) \sqrt{A}} \\
\times \operatorname{artanh}\left[\sqrt{\frac{\epsilon}{A}} \tanh \left(\sqrt{\epsilon}\left(t-t_{0}\right) / \rho\right)\right],
\end{array}
$$




$$
\frac{d \phi(t)}{d t}=\frac{-\omega \epsilon}{\cosh \left(r_{0} / \rho\right)\left[(A-\epsilon) \sinh ^{2}\left(\sqrt{\epsilon}\left(t-t_{0}\right) / \rho\right)+A\right]} .
$$

In the case of the Euclidean space the angular velocity is constant whereas in the case of the Lobachevsky space the angular velocity is variable and tends to zero at $t \rightarrow$ $\infty$.

\section{CONCLUSION}

In conclusion several additional remarks should be given. It can be shown that the formula (21) for trajectories in the form $F(r, \phi)=0$ describes not only the motions on cylindrical surfaces, shifted or not with respect to the initial axis $z$, but also describes motions infinite in radial variable $r$. So, there exist two different classes of trajectories, finite and infinite in radial variable.

Besides, it can be demonstrated that the potential of the magnetic field (4) is invariant with respect to shifts in the Lobachevsky space in the plane perpendicular to the axis $z$. This symmetry underlies the structure of two families of possible solutions of the equations of motion, providing us with a transitivity group acting within two classes of motions.

All results can be extended to a spherical Riemann model $S_{3}$ on the base of the metric

$$
\begin{array}{r}
d S^{2}=c^{2} d t^{2}-\cos ^{2}(z / \rho) d r^{2} \\
-\rho^{2} \cos ^{2}(z / \rho) \sin ^{2}(r / \rho) d \phi^{2}-d z^{2}, \\
z \in[-\pi / 2,+\pi / 2], \quad r \in[0,+\pi], \quad \phi \in[0,2 \pi]
\end{array}
$$

and a potential for a magnetic field in $S_{3}$ according to

$$
A_{\phi}=\rho^{2} B[\cos (r / \rho)-1] .
$$

\section{ACKNOWLEDGMENTS}

The authors are grateful to E. A. Tolkachev for valuable comments and useful discussion. This work has been supported by the Belarusian Republican Foundation for Fundamental Research (Project No. F09D - 009).
[1] L. D. Landau and E. M. Lifshitz, The classical theory of fields, Pergamon Press, Oxford, 1975.

[2] L. D. Landau and E. M. Lifshitz, Quantum mechanics, non-relativistic theory, Pergamon Press, Oxford, 1977.

[3] L. D. Landau, Z. Phys. 64 629-637 (1930).

[4] A. A. Bogush, V. M. Red'kov and G. G. Krylov, Doklady of the National Academy of Sciences of Belarus 53 45-51 (2009).

[5] A. A. Bogush, V. M. Red'kov and G. G. Krylov, Nonlinear Phenomena in Complex Systems 11 403-416 (2008).

[6] M. N. Olevskiy, Matem. Sbornik 27 379-426 (1950). 